\title{
An Analysis of Factors Contributing to the Development of Total Parenteral Nutrition-Induced Cholestasis
}

\author{
Robert A. Drongowski, M.A. And ARnold G. CoRan, M.D. \\ From the Section of Pediatric Surgery, Mott Children's Hospital, University of Michigan Medical Center, Ann Arbor, Michigan
}

\begin{abstract}
The risk of developmental of total parenteral nutrition (TPN)-associated cholestatic jaundice in neonates receiving intravenous hyperalimentation is high. Numerous factors have been cited as contributing to TPN cholestasis; however, the exact etiology remains obscure. This retrospective study was undertaken in order to identify any factors which might contribute to this syndrome.

The hospital records of 172 neonates requiring TPN for a minimum of 1 week were reviewed. In addition, a subgroup of 32 infants requiring TPN for a minimum of 7 weeks was also examined. Cholestasis was defined as a direct serum bilirubin greater than $2.0 \mathrm{mg} / \mathrm{dl}$ during the course of TPN therapy.

Significant factors for the development of cholestasis in both groups $(n=172, n=32)$ included: number of operations ( 2.56 vs. 1.08, $p=0.0000$ ), the number of days the patients received antibiotics ( 40.3 us $12.9, p=0.0000)$, and delayed start of enteral feedings (33.8 vs 14.1, $p=0.0000$ ).

Fifteen of the 32 patients who received TPN for at least 7
\end{abstract}

weeks did not develop cholestasis. In this subgroup $(n=32)$, there were no differences in birth weight, gestational age, days from birth to the start of TPN, or respiratory distress between those who developed cholestasis and those who remained anicteric. In contrast, there were significant differences between the cholestasis and noncholestasis groups in number of operations ( 13 vs $6, p=0.0407$ ), and days until enteral feedings were started (33.1 vs $18.9, p=0.0289)$.

This study suggests that the factor(s) contributing to the development of 'TPN-associated cholestasis are likely multifactorial. There appears to be a direct correlation between increasing severity of cholestatic jaundice and duration of TPN. This review does add a new parameter to the various causative factors suggested, namely the number of operative procedures. This new variable could be related to the stress of surgery itself or to the repeated administration of anesthetic agents. (Journal of Parenteral and Enteral Nutrition 13:586-589, 1989)
Despite attempts by numerous investigators to determine the etiology of total parenteral nutrition (TPN)associated hepatic dysfunction, the exact cause of TPN induced cholestasis remains obscure. The risk of developing TPN-associated cholestatic jaundice in neonates requiring intravenous (iv) hyperalimentation remains high. ${ }^{1-3}$ Although this condition usually resolves with time after discontinuation of the TPN, ${ }^{4-7}$ a small percentage of cases remain intractable ${ }^{8}$ and/or progress to severe hepatic dysfunction and death. ${ }^{9-11}$

Merritt, ${ }^{12}$ Seashore,${ }^{13}$ and Farrell and Balistreri, ${ }^{14}$ in separate reviews have cited the various factors thought to contribute to TPN cholestasis. These include the following: 1) clinical components: low-birth weight, prematurity, duration of TPN, immature enterohepatic circulation, intestinal microflora, bacterial sepsis; and 2) metabolic components: energy overload, increased levels of toxic bile salts, enteral starvation, amino acid composition of infusate, excess or deficiency of specific amino acids, and mineral/trace element excesses or deficiencies.

This study was designed to examine pertinent demographic, clinical, and nutritional components in a large population of neonates requiring TPN in order to refute or confirm findings reported in earlier studies and to determine if additional factors contribute to this syndrome.

Received for publication, October 31, 1988.

Accepted for publication, February 13, 1989.

Reprint requests: Arnold G. Coran, MD, Prof. of Surgery, F7516 Mott Children's Hospital, University of Michigan, Ann Arbor, MI 48109-4025.

\section{METHODS}

The hospital records of 172 neonates requiring TPN for a minimum of 7 days wee retrospectively reviewed. Descriptive data as well as total input an output for each 24-hr period the infant received intravenous nutrition were recorded. A subpopulation of these patients $(\mathbf{n}=$ 32) received TPN for a minimum of 7 weeks. These patients were additionally analyzed as a separate group since the length of administration of the TPN was quite long.

All patients were begun on a peripherally administered TPN program of glucose, crystalline amino acids, and either a safflower oil emulsion (Liposyn, Abbott Laboratories) or a soybean oil emulsion (Intralipid, Baxter Laboratories). Patients requiring long-term TPN (greater than 3-4 weeks were switched to centrally administered TPN). The solutions for centrally administered TPN consisted of $25 \%$ glucose and $3.5 \%$ crystalline amino acids, whereas the peripheral regimen consisted of $12.5 \%$ glucose and $2.5 \%$ crystalline amino acids. The fat emulsion provided a maximum of $40 \%$ of the daily caloric input for both the centrally and peripherally administered TPN.

Variables analyzed included: birth weight, gestational age at birth, number of operations, days on iv antibiotics, serum biochemistries, and mean weekly caloric intake divided into the following categories: lipid calories $/ \mathrm{kg}$, protein calories $/ \mathrm{kg}$, carbohydrate calories $/ \mathrm{kg}$, total calories $/ \mathrm{kg}$, and nonprotein calories $/ \mathrm{kg}$.

Cholestasis was defined as a direct bilirubin greater 
than $2.0 \mathrm{mg} / \mathrm{dl}$ during the course of TPN therapy. Other cause of an elevated serum bilirubin were excluded in all patients analyzed $(\mathrm{n}=172)$.

Statistical analysis was performed using the student's $t$-test and chi-square analysis with $p$-values less than 0.05 considered statistically significant.

\section{RESULTS}

There were 92 boy (53.5\%) and 80 girl infants (46.5\%) in the study cohort. A total of 74 patients $(43.0 \%)$ underwent a minimum of one major surgical procedure. Of the 172 patients studied, $26(15.1 \%)$ developed cholestasis.

There were no significant differences in birth weight ( $p=0.6089)$ or gestational age $(p=0.7594)$ between those infants developing cholestasis and those who did not. However, the number of operations (2.56 in the cholestasis group vs 1.08 in the noncholestasis group) as well as the number of days the patients received intravenous antibiotics (40.3 in the cholestasis group vs 12.9 in the noncholestasis group) were significantly different ( $p=0.0000$ in each category). In addition, the infants who developed cholestasis waited 33.8 days (mean) before the start of enteral feedings compared to 14.1 days (mean) for the patients without cholestasis $(p=0.0000)$ (Table I).

The underlying diseases and clinical course in the subgroup of newborns $(n=32)$ who received TPN for a minimum of 7 consecutive weeks is similar. Seventeen of these patients developed cholestasis within this time frame (group 1), while 15 remained anicteric (group 2). There were major anomalies in these patients in the gastrointestinal, cardiac, pulmonary, and nervous systems.

The variables which proved significant for the development of cholestasis in the subgroup of patients who received TPN for a minimum of 7 weeks were as follows: number of patients undergoing surgery, (13 vs $6, p=$ 0.0407 ) and days from birth to the start of enteral feedings (33.1 us 18.9, $p=0.0289$ ) in the cholestasis and noncholestasis groups, respectively. The actual number of operations (2.69 vs 1.33) and the number of days receiving iv antibiotics (44.6 vs 26.4) were close to being statistically significantly different $(p=0.0632$ and $p=$ 0.0602 , respectively). In contrast, there were no significant differences between the cholestasis and noncholestasis groups in birth weight, gestational age, and the number of days from birth to start of TPN ('Table II).

In addition, in this sub-group of newborns, there were no significant differences between those with cholestasis (group 1) and those without cholestasis (group 2) with

TABLE I

Variables related to $T P N$-induced cholestasis $(n=172)$

\begin{tabular}{lccc}
\multicolumn{1}{c}{ Variable } & $\begin{array}{c}\text { Cholestasis } \\
\text { group }\end{array}$ & $\begin{array}{c}\text { Noncholestasis } \\
\text { group }\end{array}$ & p-value \\
\hline Birth weight & 1.46 & 1.56 & 0.6089 \\
Gestational age & 30.6 & 31.0 & 0.7594 \\
Number of operations per patient & 2.56 & 1.08 & 0.0000 \\
Days receiving antibiotics & 40.3 & 12.9 & 0.0000 \\
Days from birth to enteral feed- & 33.8 & 14.1 & 0.0000 \\
$\quad$ ings & & & \\
\hline
\end{tabular}

respect to the following TPN components expressed as the mean weekly value per kilogram administered: protein calories (Fig. 1), glucose calories (Fig. 2), lipid calories (Fig. 3), total calories (Fig. 4) or nonprotein calories (Fig. 5).

The development of cholestasis with increasing duration of TPN is presented in Figure 6. Cholestasis did not develop in any patients who received TPN for only 1 week. In contrast, $100 \%$ (all seven) of the patients who received TPN for a minimum of 12 weeks developed cholestatic jaundice.

The mean value of the highest direct bilirubin levels for the patients who received TPN for 1 to 6 weeks $(\mathrm{n}=$ 9), 7 to 10 weeks $(n=9)$, and more than 11 weeks $(n=$ 8 ) is presented in Figure 7. There is an increasing mean

TABLE II

Variables related to onset of cholestasis in patients receiving TPN for 7 consecutive weeks $(n=32)$

\begin{tabular}{lccc}
\hline \multicolumn{1}{c}{ Variable } & $\begin{array}{c}\text { Cholestasis } \\
\text { group }(\mathrm{n}=17)\end{array}$ & $\begin{array}{c}\text { Noncholestasis } \\
\text { group ( } \mathrm{n}-15)\end{array}$ & p-value \\
\hline $\begin{array}{l}\text { Days from birth to start of } \\
\quad \text { enteral feedings }\end{array}$ & 33.1 & 18.9 & 0.0289 \\
$\begin{array}{l}\text { Number of operations per } \\
\quad \text { patient }\end{array}$ & 2.69 & 1.33 & 0.0632 \\
$\begin{array}{l}\text { Days receiving antibiotics } \\
\text { Birth weight }\end{array}$ & 44.6 & 26.4 & 0.0602 \\
Gestational age & 1.46 & 1.17 & 0.2683 \\
Days from birth to start of & 30.8 & 29.5 & 0.4257 \\
$\quad$ TPN & 5.44 & 4.93 & 0.8308 \\
Surgery & & & \\
$\quad$ Yes & 13 & 6 & 0.0407 \\
$\quad$ No & 4 & 9 & \\
\hline
\end{tabular}

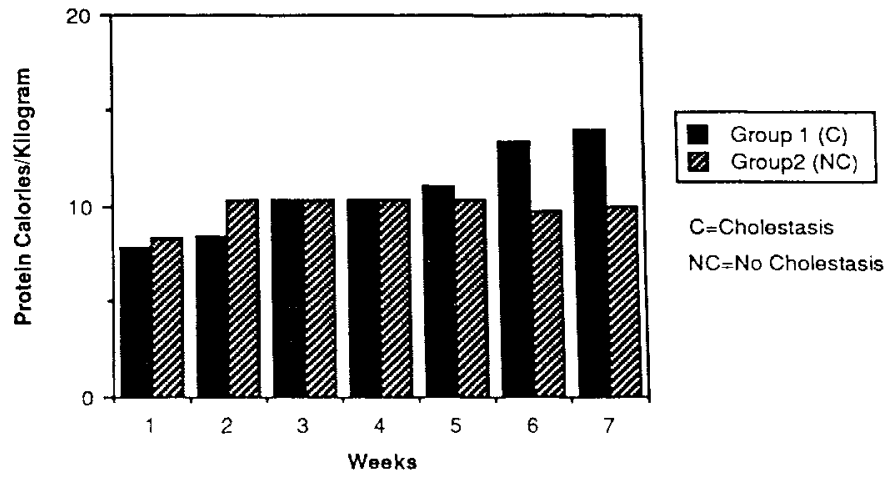

FIG. 1. Protein calories (mean) administered per week.

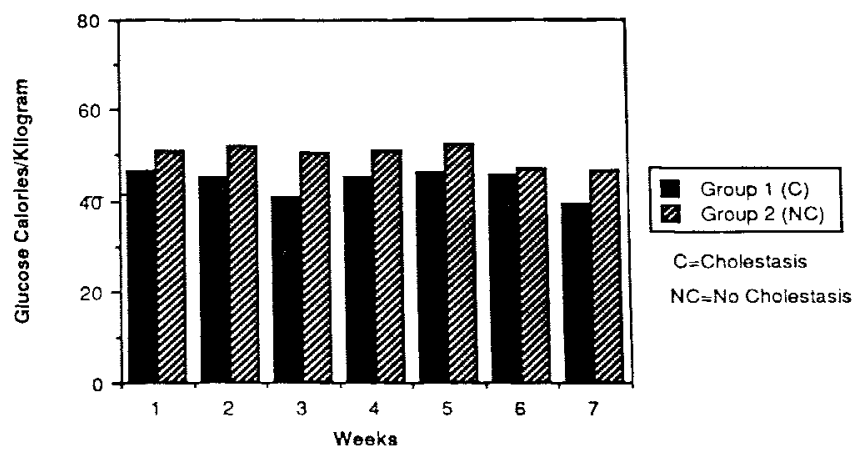

Fig. 2. Glucose calories (mean) administered per week. 


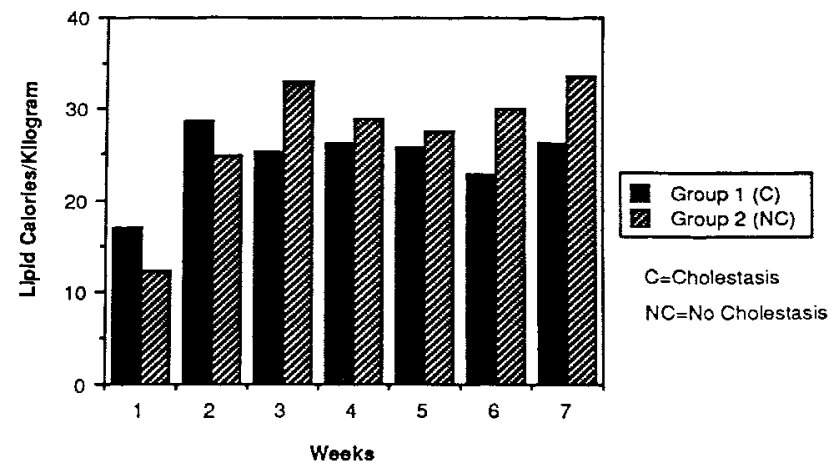

FrG. 3. Lipid calories (mean) administered per week.

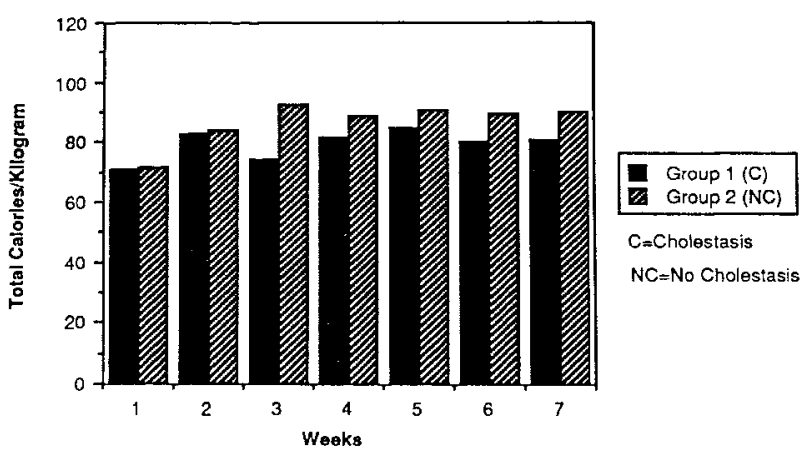

FIG. 4. Total calories (mean) administered per week.

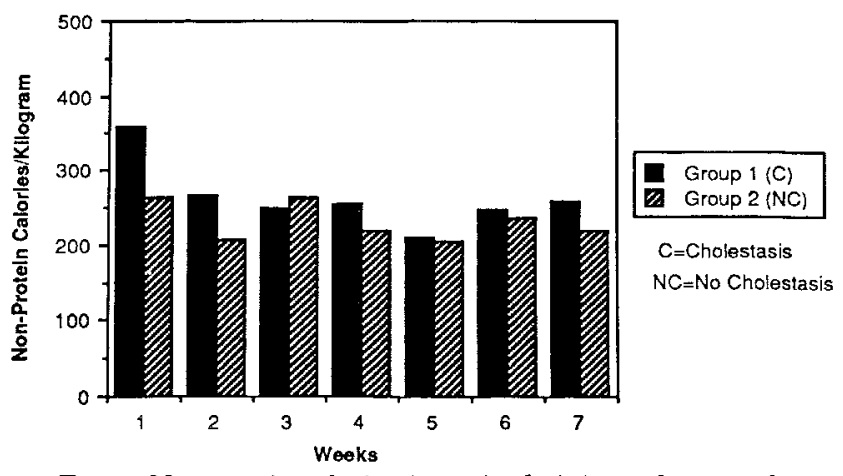

FIG. 5. Nonprotein calories (mean) administered per week.

bilirubin level with increasing duration of TPN, $4.21 \pm$ $1.63,4.91 \pm 1.44$, and $5.56 \pm 1.61$, respectively.

\section{DISCUSSION}

The reported incidence of TPN-associated cholestasis in infants receiving iv nutrition varies from 7 to $42 \% .^{15,16}$ In this study, $15.1 \%$ of the total population examined developed cholestatic jaundice, defined as a direct bilirubin equal to or greater than $2.0 \mathrm{mg} \%$ during the course of iv nutrition. As previously reported, sepsis ${ }^{17}$ and number of operations ${ }^{1,2}$ were risk factors for the development of this condition in our series. However, low birth weight, ${ }^{18,19}$ prematurity, ${ }^{20}$ respiratory distress, ${ }^{15}$ or quantity of protein, ${ }^{21}$ glucose,${ }^{16}$ lipid, ${ }^{22}$ and total calories infused were not significant risk factors associated with TPN-cholestasis in this series ( $v s$ most of the others reported in the literature). The previously reported re-

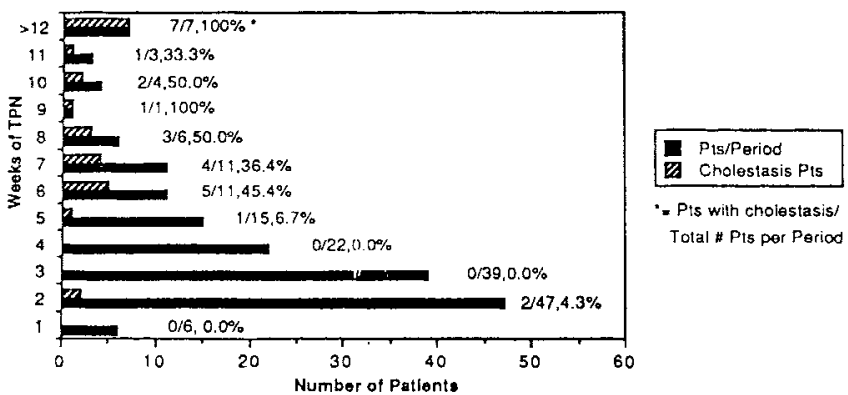

FIG. 6. Cholestasis and noncholestasis patient distribution vs weeks of TPN therapy.

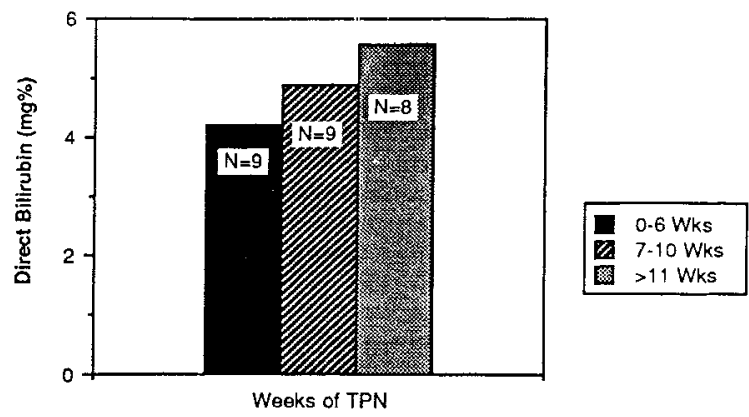

FIG. 7. Highest direct bilirubin levels (mean) during TPN therapy.

lationships probably reflect the fact that more newborns who are premature, have low birth weight and who experience respiratory distress require TPN in comparison with other neonates and probably undergo more operations than other newborns. Most importantly, this study argues against the likelihood of the TPN solution per se contributing to cholestasis, ${ }^{6}$ since both the group 1 and group 2 infants received similar solutions for three and one-half times the minimal interval ( 2 weeks) considered as necessary for the development of cholestasis ${ }^{23,24}$ an observation which has not been previously reported.

This study, like others, suggests that the factor(s) contributing to the development of TPN-associated cholestasis are likely multifactorial, and as yet, not completely determined. There appears to be a direct correlation between increasing severity of cholestatic jaundice (increasing serum levels of direct bilirubin) and duration of TPN. However, this study does add a new parameter to the various causative factors suggested, namely the number of operative procedures. This new variable could be related to the stress of surgery itself or to the repeated administration of anesthetic agents. If one further postulates that an immature hepatobiliary system is initially subjected to the stress of birth, followed shortly thereafter by the stress of surgery, then it is easy to imagine the development of hepatic dysfunction and resulting cholestasis.

\section{REFERENCES}

1. Ginn-Pease ME, Pantalos D, King DR: TPN-associated hyperbilirubinemia: A common problem in newborn surgical patients. $J$ Pediatr Surg 20:436-439, 1985 
2. Bell RL, Ferry GD, Smith EO, et al: Total parenteral nutritionrelated cholestasis in infants. JPEN 10:356-359, 1985

3. Sheard NF, Kleinman RE: TPN cholestasis in premature infants: The role of parenteral nutrition solutions: Pediatr Ann 16:246252,1987

4. Klein GL, Rivera D: Adverse metabolic consequences of total parenteral nutrition. Cancer 55:305-308, 1985

5. Dahms BB, Halpin TC, Jr: Serial liver biopsies in parenteral nutrition-associated cholestasis of early infancy. Gastroenterology $81: 136-144,1981$

6. Postuma R, Trevenen CL: Liver disease in infants receiving total parenteral nutrition. Pediatrics 63:110-115, 1979

7. Brown MR, Putnam TC: Cholestasis associated with central intravenous nutrition in infants. NY State J Med 78:27-30, 1978

8. Cooper A, Ross III AJ, O-Neill JA, et al: Resolution of intractable cholestasis associated with total parenteral nutrition following biliary irrigation. J Pediatr Surg 20:772-774, 1985

9. Cooper A, Betts JM, Pereira GP, et al: Taurine deficiency in the severe hepatic dysfunction complicating total parenteral nutrition. J Pediatr Surg 19:462-466, 1984

10. Hodes JE, Grosfeld JL, Weber TR, et al: Hepatic failure in infants on total parenteral nutrition (TPN): Clinical and histopathologic observations. J Pediatr Surg 17:463-468, 1982

11. Cohen C, Olsen MM: Pediatric total parenteral nutrition. Arch Pathol Lab Med 105:152-156, 1981

12. Merritt RJ: Cholestasis associated with total parenteral nutrition. J Pediatr Gastroenter Nutr 5:9-22, 1986

13. Seashore JH: Metabolic complications of parenteral nutrition in infants and children. Surg Clin North Am 60:1239-1252, 1980

14. Farrell MK, Balistreri WF: Parenteral nutrition and hepatobiliary dysfunction. Clin Perinatol 13:197-212, 1986

15. Beale EF, Nelson RM, Bucciarelli RL, et al: Intrahepatic cholestasis associated with parenteral nutrition in premature infants. Pediatrics 64:342-347, 1979

16. Vileisis RA, Inwood RJ, Hunt CE: Prospective controlled study of parenteral nutrition-associated cholestatic jaundice: Effect of protein intake. J Pediatr 96:893-897, 1980

17. Manginello FP, Javitt NB: Parenteral nutrition and neonatal cholestasis. J Pediatr 94:296-298, 1979

18. Sondheimer JM, Bryan H, Andrews W, et al: Cholestatic tendencies in premature infants on and off parenteral nutrition. Pediatrics 62:984-989, 1978

19. Rodgers BM, Hollenbeck JI, Donnelly WH, et al: Intrahepatic cholestasis with parenteral alimentation. Am J Surg 131:149-155, 1976

20. Pereira GR, Sherman MS, DiGiacomo J, et al: Hyperalimentationinduced cholestasis. Am J Dis Child 135:842-845, 1981

21. Bernstein J, Chang $\mathrm{CH}$, Brough $\mathrm{AJ}$, et al: Conjugated hyperbilirubinemia in infancy associated with parenteral alimentation. $J$ Pediatr 90:361-367, 1977

22. Salvian AJ, Allardyce DB: Impaired bilirubin secretion during total parenteral nutrition. J Surg Res 28:547-555, 1980

23. Greenlaw $\mathrm{CW}$ : Liver enzyme elevation with total parenteral nutrition. Drug Intell Clin Pharm 14:702-709, 1980

24. Lowry SF, Brennan MF: Abnormal liver function during parenteral nutrition: Relation to infusion excess. J Surg Res 26:300-307, 1979 